\title{
Stimulation of the hypothalamic-pituitary-adrenal axis with corticotropin releasing hormone in patients with psoriasis
}

\author{
Evangelos Karanikas, Faidon Harsoulis, Ioannis Giouzepas, Ioannis Griveas
}

Departments of Dermatology, Psychiatry and Endocrinology, Medical School, Aristotle University of Thessaloniki, Greece

\begin{abstract}
OBJECTIVE: Psychocutaneous diseases constitute a large proportion of psychosomatic disorders, with psoriasis being one of the most typical cases. Though alteration of HypothalamicPituitary-Adrenal (HPA) axis function has been suggested as underlying several psychiatric disorders and psychosomatic diseases, there is little evidence of reduced response of the HPA axis in psoriasis after psychosocially induced laboratory stress. The aim of the study was to investigate any alteration of the neuroendocrine profile of psoriatic patients. DESIGN: The psoriatic patients $(n=24)$ and the same number of matched controls underwent a CRH test which consisted of $100 \mu \mathrm{g}$ h-CRH IV infusion and drawing of blood samples at 0 min and at 15, 30 and 60 min post h-CRH for measurement of plasma ACTH and cortisol concentration. RESULTS: Mean plasma ACTH and cortisol levels in both groups increased during the 60min CRH test without significant difference. The total secretion of plasma ACTH and serum cortisol estimated as Area Under the Curve did not show significant difference between the groups either. CONCLUSIONS: Contrary to previous studies no particular neuroendocrine profile of HPA axis responsiveness was identified in psoriatic patients.
\end{abstract}

Key words: ACTH, CRH, Cortisol, Hypothalamus-Pituitary-Adrenal axis, Psoriasis, Stress

\section{INTRODUCTION}

Psoriasis constitutes one of the most illustrative examples of the close relation between exacerbations of cutaneous lesions and the psychopathologic burden of the patients. Numerous studies have attempted to identify a specific personality profile of the psoriatic patient, ${ }^{1,2}$ but it remains unclear whether different

Address for correspondence:

Evangelos Karanikas, 10 Kleanthous Str., 54642, Thessaloniki, Greece, Tel: +30 6977313243, Fax: +30 2310381010 ,

e-mail: epkarani@yahoo.com

Received 10-02-07, Revised 10-07-07, Accepted 20-08-07 psychological features precede the cutaneous flareups and precipitate relapses of the disease or are the consequence of suffering from a chronic, symptomatic, disfiguring illness.

Furthermore, many studies ${ }^{3-10}$ have focused on the relation between stressful life events and exacerbation of the psoriatic disease. According to one of them, ${ }^{5}$ psoriatic patients whose cutaneous condition is closely connected to their exposure to stressful life events were reported to be up to $80 \%$.

On the other hand, the research output investigating the psychophysiology of psoriasis, especially 
within the neuroendocrine framework, is minimal. The Hypothalamic-Pituitary-Adrenal (HPA) axis comprises one of the two main regulators of the stress system, with the Locus Ceruleous-SympathoAdreno-Medullary (SAM) axis being the other. ${ }^{11,12}$ Alterations of its function have been suggested as implicated in a number of sheer psychiatric disorders and psychosomatic diseases as well. In particular, in Major Depressive Disorder (MDD), a hyporeactivity of the HPA axis after stimulation has been proposed, ${ }^{13,14}$ as evidenced by blunted ACTH response in the CRH test. In another psychiatric disorder, Post Traumatic Stress Disorder (PTSD), the application of the CRH test exhibited blunted plasma ACTH response, ${ }^{15}$ suggesting a possible downregulation of the pituitary's CRH receptors that could indicate either a chronic hypothalamic $\mathrm{CRH}$ hypersecretion and/or an increase of circulating glucocorticoids negative feedback action. Rupprecht et $\mathrm{al}^{16}$ investigated the HPA axis function of patients with atopic dermatitis with application of the CRH test.

The aforementioned studies were conducted with regard to the function of the HPA axis in contrast with the recently proposed ${ }^{17,18}$ concept of a skin stress response system coordinated by a local cutaneous HPA axis-like system. This suggestion has been based on findings by immunohistochemical studies ${ }^{19}$ that demonstrated increased CRH and CRH-Receptors staining throughout the epidermis and dermal vasculature in patients with active psoriasis as well as induction of skin vascular permeability by peripheral $\mathrm{CRH}$ through a neurotensin-dependent process. ${ }^{20}$

To our knowledge, there are 6 studies ${ }^{21-26}$ involving neuroendocrine parameters of the systemic HPA axis in psoriatic patients, which do not implicate the locally functioning cutaneous HPA-like system. The $\mathrm{CRH}$ test has not been used in any of them. The aim of the present study was to further elucidate the systemic HPA axis function in psoriasis via application of the CRH test.

\section{SUBJECTS AND METHODOLOGY}

\section{Patients}

The study involved the participation of 24 patients who visited the $\mathrm{A}^{\prime}$ Clinic of Dermatological and Venereological Diseases of the Aristotelian University of Thessaloniki on a primarily outpatient basis. They suffered from psoriasis which had been diagnosed either on a clinical basis (the diagnosis had been made by two clinicians) or by histopathological findings. Eleven (11) patients ( $46 \%$ of the total sample) were males and thirteen (13) (54\% of the total sample) females. The mean \pm sem (standard error of mean) age of the patients was $51.29 \pm 3.59$ years.

From the group of patients, three ( 2 males and one female) finally had to be admitted to the hospital. The mean duration of the disease since it was initially diagnosed has been recorded in years mean \pm sem, $6.29 \pm 1.90$ and, according to the subjective reports of the patients, the mean duration of the current psoriatic episode was estimated as well in months mean \pm sem, $6.28 \pm 1.61$ [n:18 (6 gave insufficient reports)].

Twenty-four healthy volunteers were also recruited for the study: 11 (46\%) were males and $13(54 \%)$ were females with mean age, $\mathrm{x} \pm$ sem, $43.42 \pm 2.35$ years. The groups under study were matched in terms of gender and age. Age of psoriatics and controls were not significantly different (p:0.075).

The protocol of the study was approved by the ethical committee of both the Medical School of the Aristotelian University and the Hospital of Dermatological and Venereological Diseases of Thessaloniki. All the participants gave their written consent after having been told the exact procedure.

The inclusion and exclusion criteria for both healthy volunteers and psoriatics were: 1 ) age between 18-60,2) participation in at least 10 years of the state educational system, 3) exclusion of major pathological conditions (cardiopulmonary, renal failure, cancer, cerebrovascular episode, etc), 4) exclusion of surgical operation during the last 6 months, 5 ) exclusion of alcoholism or heavy drinking (over 60 drinks per month), 6) exclusion of use of drugs at least 2 weeks prior to the study, 7) exclusion of use of medication known for its influence on the immune system (anti-inflammatory, antibiotics, psychiatric, antihistaminic, $\beta$-blockers) at least 2 weeks prior to the study, 8) exclusion of active bacterial or viral infection, 9) exclusion of major psychiatric disorders, such as MDD, known for their impact on HPA axis function, through psychiatric evaluation.

Psoriatic patients should had been off drugs such as 
corticosteroids and other immunomodulating agents like methotrexate, cyclophosphamide, cyclosporine, acitretin and PUVA for at least 3 months prior to the study and should likewise be off even external application of corticosteroids for at least 1 week prior to participation in the study.

The flare-ups of the psoriatic lesions should be either initially diagnosed or recently relapsed so that the active state of the psoriatic condition may be ensured.

Of the psoriatic group, 13 had the plaque type, 5 the palmoplantaric type, 3 at the scalp, 2 the generalized and one the erythrodermal type, while the extent of psoriatic lesions, according to the rule of "nine" used by the clinicians, was: small in $16(67 \%)$ patients ( $<10 \%$ of body surface), medium in $7(29 \%)$ patients $(10 \%<. .<20 \%$ of body surface) and extensive in one $(4 \%)$ patient ( $>20 \%$ of body surface).

Of the psoriatic group, 11 patients were under treatment with external application of corticosteroids until 1 week prior to the study, 8 reported hypertension under treatment (except $\beta$ blockers), one chronic gastritis and one phlebitis of the legs.

Of the group of the healthy volunteers, 2 reported hypertension under treatment.

\section{Procedure}

All the participants in the study had undergone general hematological and biochemical tests with normal findings.

\section{CRH test}

The procedure began at 08:00. The volunteer remained supine for 10 minutes. The reduction of the display at external stimuli was taken into account. A heparinised cannula was inserted into a forearm vein to allow repeated drawings of blood samples. The volunteer remained another 10 minutes so that the stress of the puncture be reduced and not influence the hormones under study. Subsequently, after a first blood sample had been drawn at time 0 minutes, 100 $\mu \mathrm{g}$ of h-CRH (Ferring, Germany) dissolved in $1 \mathrm{ml}$ saline $(0.9 \%)$ was infused intravenously for 5 seconds. The procedure was completed with consecutive collections of blood samples at 15, 30 and $60 \mathrm{~min}$ from the initial blood sample. Plasma was collected in prechilled tubes with EDTA and within 2 hours the samples were centrifuged at $4^{\circ} \mathrm{C}$ and $3000 \mathrm{rpm}$ for 10 minutes. Afterwards they were stored at $-80^{\circ} \mathrm{C}$ until assayed.

During the procedure of the CRH test no adverse effect was recorded except for a transient upper and facial flash, which disappeared during the first few minutes of the test.

\section{Hormone Assays}

Plasma ACTH was measured by dual antibody immunoradiometric assay using a commercial kit (BRAHMS DYNOtest ACTH Hennigsdorf, Germany). The intra-assay variability was $7 \%$ at an average concentration of $30 \mathrm{pg} / \mathrm{ml}$ and the inter-assay variability was $<8 \%$. The analytical and functional sensitivity of the assay were 1.2 and $2.4 \mathrm{pmol} / \mathrm{l}$, respectively. The normal values of ACTH plasma concentration of the particular method for morning measurements were $10-60 \mathrm{pg} / \mathrm{ml}$ and the conversion factor $\mathrm{pg} / \mathrm{ml}$ : $\mathrm{pmol} / \mathrm{l} \times 4.541$.

The serum cortisol was measured with a radioimmunoassay kit of DSL-2100 ACTIVE Cortisol RIA, Texas, USA. The expected normal morning values were $9-23 \mu \mathrm{g} / \mathrm{dl}$. The detection limit was $0.5 \mu \mathrm{g} / \mathrm{dl}$. The intra-assay and inter-assay coefficient of variation were $5 \%$ and $9 \%$ at an average concentration of $19 \mu \mathrm{g} / \mathrm{dl}$.

\section{Data Analysis}

Comparisons of plasma ACTH and serum cortisol concentrations were based on the mean of the respective group of values at $0,15,30$ and $60 \mathrm{~min}$ for each subject, expressed as means \pm sem. To further evaluate the hormones secretion during the 60 minutes of the $\mathrm{CRH}$ test, the Areas Under the response Curve, corrected for baseline (AUC) using the trapezoidal integration, was estimated. The data were analysed using Independent samples t-test and Pearson's product-moment correlation. The level of statistical significance used in every comparison was $5 \%(\mathrm{p} \leq 0.05)$.

\section{RESULTS}

\section{CRH test}

After $\mathrm{CRH}$ infusion, the mean \pm sem $\mathrm{ACTH}$ plas- 
ma values in $\mathrm{pg} / \mathrm{ml}$ for psoriatics were $21.71 \pm 4.20$, $39.37 \pm 6.47,40.24 \pm 7.84$ and $32.00 \pm 6.32$ and for the controls were $16.10 \pm 2.20,37.28 \pm 5.42,35.26 \pm 4.94$ and $27.43 \pm 3.53$, measured at $0,15,30$ and $60 \mathrm{~min}$, respectively. The $t$ test between the mean values at 0 (p:0.233), 15 (p:0.805), 30 (p:0.590) and 60 (p:0.526) min did not show significant differences (Figure 1).

Moreover, the total secretion of ACTH during the $\mathrm{CRH}$ test was estimated in both groups as Area Under the Curve (AUC) according to the trapezoid rule. The mean \pm sem total secretion of $\mathrm{ACTH}$ as AUC for psoriatics was $2.062 \pm 0.379 \mathrm{pg} \times 1000 \times \mathrm{min} / \mathrm{ml}$ and $1.804 \pm 0.241$ for the controls without significant difference, t:-0.580, df:45, p:0.565 (Figure 1).

The same procedure was followed for serum cortisol values during the $\mathrm{CRH}$ test. Specifically, the mean \pm sem cortisol values for psoriatics, in $\mu \mathrm{g} / \mathrm{dl}$, at $0,15,30$ and $60 \mathrm{~min}$ were $18.03 \pm 1.98,18.59 \pm 1.56$, $20.51 \pm 1.72$ and $18.59 \pm 1.33$, respectively, while the corresponding cortisol values for healthy volunteers were $16.94 \pm 1.22,18.83 \pm 1.45,19.8 \pm 1.73$ and $20.06 \pm 1.34 \mu \mathrm{g} / \mathrm{dl}$. The $\mathrm{t}$ test between the mean values at 0 (p:0.639), 15 (p:0.914), 30 (p:0.775) and 60 (p:0.442) min did not show significant difference between the two groups (Figure 2).

The mean \pm sem total secretion of cortisol during the $\mathrm{CRH}$ test estimated as AUC for the psoriatics and healthy controls was $1.155 \pm 0.087$ and $1.156 \pm 0.080$

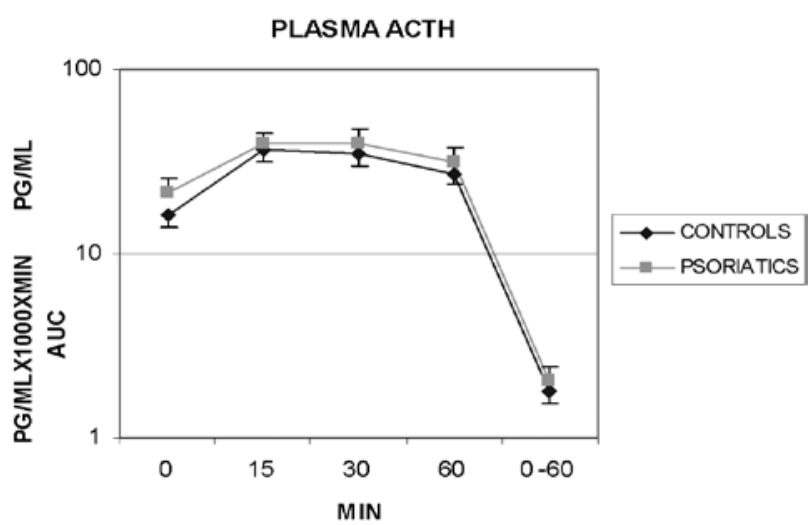

Figure 1. Algorithmic graph of mean \pm sem ACTH plasma values, in $\mathrm{pg} / \mathrm{ml}$, in the control and psoriatic groups, during the $\mathrm{CRH}$ test, at $0 \mathrm{~min}$ and at 15,30 and $60 \mathrm{~min}$ and secretion of plasma ACTH during $60 \mathrm{~min}$, as AUC, in $\mathrm{pg} \times 1000 \times \mathrm{min} / \mathrm{ml}$. $\mathrm{ACTH}(\mathrm{pg} / \mathrm{ml})$ : To convert to SI units multiply by 0.22 . $\mu \mathrm{g} \times 1000 \times \mathrm{min} / \mathrm{dl}$, respectively, without significant difference (p:0.991).

Among the psoriatic group, serum ACTH as AUC did not demonstrate any statistically significant correlation with the age (p:0.162), gender (p:0.236), extent of the cutaneous lesions (p:0.090), duration of the disease (p:0.385) and the current episode (p:0.524).

\section{Sub-grouping of psoriatic patients}

To further explore if the groups under study (controls and psoriatics) could be divided into subgroups of responders/non-responders, a well established threshold ${ }^{27}$ (doubling the basal ACTH level at 0 during the $60 \mathrm{~min}$ of the CRH test) was used. Those whose ACTH values exceeded the aforementioned threshold formed the responders subgroup (18 out of 24 from the healthy group and 12 out of 23 from the psoriatic group; in one psoriatic patient no data were collected due to technical reasons), in contrast to the non-responders (6 controls and 11 psoriatics) who did not exceed the cut-off. The Chi Square analysis between the subgroups did not reach statistical significance (p: 0.149). Furthermore, the large variance of the $\mathrm{ACTH}$ values in both groups during the $\mathrm{CRH}$ test is a basic statistical factor that by definition suggests an indeterminancy or non-existence of a threshold (other than the aforementioned one ${ }^{27}$ ) that could divide the psoriatics into subgroups of responders and non-responders of the HPA axis function.

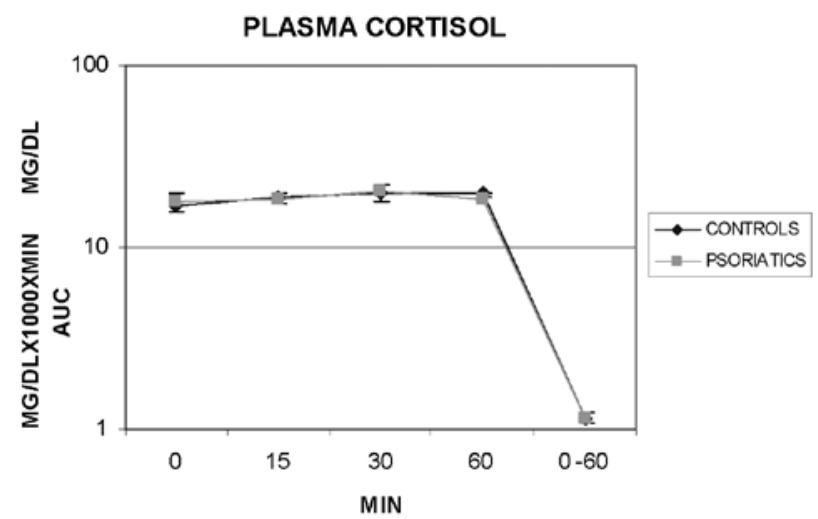

Figure 2. Algorithmic graph of mean \pm sem plasma cortisol values, in $\mu \mathrm{g} / \mathrm{dl}$, in the control and psoriatic groups, during the $\mathrm{CRH}$ test, at $0 \mathrm{~min}$ and at 15,30 and $60 \mathrm{~min}$, and secretion of plasma cortisol during $60 \mathrm{~min}$, as AUC, in $\mu \mathrm{g} \times 1000 \times \mathrm{min} / \mathrm{dl}$. Cortisol $(\mu \mathrm{g} / \mathrm{dl})$ : To convert to SI units multiply by 27.6. 
Moreover, to investigate whether the treatment with topical steroids until one week prior to the study in psoriatic patients interfered with the hormonal results of the CRH test, we performed the Tukey HSD and Scheffe post hoc tests of one-way anova analysis of the AUC ACTH among the 3 groups (controls $\mathrm{n}: 24$, psoriatics under treatment with topical steroids, $\mathrm{n}: 11$, and psoriatics on no treatment, $\mathrm{n}: 12$ ). All the $\mathrm{p}$ values were under 0.05 .

\section{DISCUSSION}

It is widely known that tissues from the nervous and cutaneous systems share common embryological origin and that there is a close relation among dermatological disorders, mental status and psychopathology. Psoriasis is one of the most typical examples of psychocutaneous disorders as the chronicity of the disease and its clinical course, with exacerbations and remissions, relate to the stressful life events and their impact on the psychological state. . $^{1-3,5,8-10}$

In the present study, the application of the $\mathrm{CRH}$ test constitutes a major advantage in relation to the exposure to psychosocial type of stressor, as it evokes less subjective results about the systemic HPA axis function.

According to the data of the few studies investigating the systemic neuroendocrine framework of psoriasis, ${ }^{21-23,25}$ there is some evidence suggesting a hyporeactive HPA axis function after stimulation. Only in one recent study ${ }^{26}$ are the findings suggestive of no alteration of the HPA axis function in psoriasis.

In particular, most of the findings of the aforementioned studies suggest that there is a decrease in serum and urine cortisol level in psoriatic patients compared with the healthy controls, during exposure to psychosocial stressor. However, there was evidence for hyper-reactivity of the SAM axis, as the level of serum and urine adrenaline was higher in psoriatics after stressor exposure. Furthermore, the two studies $^{24,26}$ involving Dexamethasone Suppression Test (DST) application, psoriatics exhibited controversial results.

In our study, the increases of the plasma ACTH and cortisol values during the $\mathrm{CRH}$ test did not show significant differences between the groups under study. These findings contradict the hypothesis for a particular neuroendocrine profile of the hyporeactivity type in psoriasis, while they support the findings of the recent study by Buske-Kirschbaum et $\mathrm{al}^{26}$ which showed no alteration of the HPA axis function either centrally with the evaluation of the ACTH level after stressor exposure or peripherally with the DST application.

Furthermore, the results (hormonal values) from the $\mathrm{CRH}$ test did not demonstrate significant correlation with the patients' demographic data such as age and gender, nor with clinical variables of the disease, such as the extent of the cutaneous lesions, total duration of the disease and duration of the present episode.

It is widely accepted ${ }^{28,29}$ that a decreased HPA axis response can mimic the glucocorticoid-deficient state and can cause increased susceptibility to autoimmune and inflammatory disease. Animal studies with Lewis rats, ${ }^{28,30}$ which possess a hypoactive HPA axis, have shown that these rodents are extremely prone to develop experimentally induced $\mathrm{TH}_{1}$ mediated states (such as arthritis, uveitis or experimental allergic encephalomyelitis). ${ }^{29}$ In regard to the psoriatic disease, it is considered to be a mainly $\mathrm{TH}_{1}$ mediated autoimmune disorder. ${ }^{31}$

The findings of the present study support the suggestion that, in a $\mathrm{TH}_{1}$ dominant inflammatory condition, the HPA axis could be normal. The controversial results of the studies aiming at evaluating the systemic HPA axis function in psoriasis suggest either the complexity of the neuroendocrine mechanisms in humans and/or the significant participation of the SAM axis in the determination of the neuroendocrine, and consequently the immune response, to the triggering factors of psoriatic flare-ups, with stress playing a predominant role. It is noteworthy that the findings of the studies ${ }^{22,23,26}$ in psoriatic patients investigating the SAM axis function unanimously suggest a hyperreactive state induced by stressful stimuli.

Additionally, the findings of the present study oppose a hypothesis of reduced number and/or affinity of the $\mathrm{CRH}$ receptors at the pituitary level that could explain the hypo-responsiveness of the HPA axis after stimulation in psoriasis proposed by other researchers. ${ }^{21-23,25}$ On the other hand, the recently sug- 
gested decreased gene expression of $\mathrm{CRH}$ receptor-1 in affected psoriatic skin has been associated with increased levels of local or systemic (serum) CRH in patients with active psoriasis. ${ }^{32}$

To summarize, the application of the CRH test in psoriatic patients for the investigation of the systemic HPA axis responsiveness did not show considerable differences compared with the control group. The divergence of the results between the present study, where a specific neuroendocrine test was applied, and the previous ones, where a psychosocial type of stressor was used, could indicate the diversity of the neuroendocrine response depending on the kind of stressful stimulation in psoriasis. Moreover, the results suggest that the HPA axis might not be the only neuroendocrine system to regulate the initiation and maintenance of the psoriatic condition. The endogeno3us complexity found to reside in the neuroendocrine mechanisms of psoriasis lays the groundwork for further studies.

\section{REFERENCES}

1. Mazzetti M, Mozzetta A, Soavi G, et al, 1994 Psoriasis, stress and psychiatry: psychodynamic characteristics of Stressors. Acta Derm Venereol Suppl (Stockh) 186: 6264.

2. Duran V, Jovanovic M, Misic-Pavkov G, et al, 1993 The effect of stress and personality structure on the onset and severity of the clinical picture of psoriasis. Med Pregl 46: 120-123.

3. Seville RH, 1977 Psoriasis and stress. Br J Dermatol 97: 297-302.

4. Gupta M, Gupta A, 1996 Psychodermatology: an update. J Am Acad Dermatol 34: 1030-1046.

5. Gupta M, Gupta A, Kikby S, et al, 1989 A psychocutaneous profile of psoriasis patients who are stress reactors, a study of 127 patients. Gen Hosp Psych 11: 166-173.

6. Harvima RJ, Viinamaki H, Harvima IT, et al, 1996 Association of psychic stress with clinical severity and symptoms of psoriatic patients. Acta Derm Venereol 76: 467-471.

7. Fava GA, Perini GI, Veller Fornasa C, 1980 Life events and psychological distress in dermatologic disorders: Psoriasis, chronic urticaria and fungal infections. Br J Med Psychol 53: 277-282.

8. Baldaro B, Brocani G, Bossi G, et al, 1989 Psoriasi. Incidenza di eventi stressanti nei sei mesi precedenti la comparsa della malattia. Med Psicosom 34: 47-51.

9. Lyketsos GC, Stratigos GC, Tawil G, et al, 1985 Hostile personality characteristics, dysthymic states and neurotic symptoms in urticaria, psoriasis and alopecia. Psychother
Psychosom 44: 122-131.

10. Payne RA, Rowland D, Payne CM, et al, 1985 Stress does not worsen psoriasis? A controlled study of 32 patients. Clin Exp Dermatol 10: 239-245.

11. Chrousos GP, Gold PW, 1992 The concepts of stress and stress system disorders: overview of physical and behavioral homeostasis. JAMA 267: 1244-1252.

12. Chrousos GP, 1992 Regulation and dysregulation of the hypothalamic-pituitary-adrenal axis: the corticotropinreleasing hormone perspective. Endocrinol Metab Clin North Am 21: 833-858.

13. Gold PW, Chrousos G, Kellner C, et al, 1984 Psychiatric implications of basic and clinical studies with corticotropin-releasing factor. Am J Psychiatry 141: 619-627.

14. Amsterdam JD, Maislin G, Winocur A, et al, 1987 Pituitary and adrenocortical responses to the ovine corticotropin releasing hormone in depressed patients and healthy volunteers. Arch Gen Psychiatry 44: 775-781.

15. Smith MA, Davidson J, Ritchie C, 1989 The corticotropin releasing hormone test in patients with PTSD. Biol Psychiatry 26: 349-355.

16. Rupprecht M, Hornstein PO, Schluter D, et al, 1995 Cortisol, Corticotropin and $\beta$-Endorphin Responses to Corticotropin-Releasing Hormone in Patients with Atopic Eczema. Psychoneuroendocrinology 20: 543-551.

17. Slominski A, Wortsman J, Pisarchik A, et al, $2001 \mathrm{Cu}-$ taneous expression of corticotrophin releasing hormone (CRH), urocortin and CRH receptors. FASEB J 15: 1678-1693.

18. Slominski A, Botchakayev V, Choudry M, et al, 1999 Is there a "skin stress system"? Ann N Y Acad Sci 885: 287-311.

19. Kono M, Nagata H, Umemura S, et al, 2001 In situ expression of corticotrophin releasing hormone $(\mathrm{CRH})$ and proopiomelanocortin (POMC) genes in human skin. FASEB J 15: 2297- 2299.

20. Donelan J, Boucher W, Papadopoulou N, et al, 2006 Corticotropin-releasing hormone induces skin vascular permeability through a neurotensin-dependent process. Proc Natl Acad Sci USA 103: 7759-7764.

21. Arnetz BB, Fjellner B, Eneroth P, Kallner A, 1991 Endocrine and dermatological concomitants of mental stress. Acta Derm Venereol Suppl (Stockh) 156: 9-12.

22. Arnetz BB, Fjellner B, Eneroth P, et al, 1985 Stress and Psoriasis: Psychoendocrine and Metabolic Reactions in Psoriatic Patients During Standardized Stressor Exposure. Psychosom Med 47: 528-541.

23. Schmidt-Ott G, Jacobs R, Jager B, et al, 1998 Stress-Induced Endocrine and Immunological Changes in Psoriasis Patients and Healthy Controls. Psychother Psychosom 67: 37-42.

24. Thaller V, Vrkljan M, Hotujac L, et al, 1999 The potential role of hypocortisolism in the pathophysiology of PTSD and psoriasis. Coll Antropol 23: 611-619.

25. Richards HL, Ray DW, Kirby B, et al, 2005 Response of the hypothalamic-pituitary-adrenal axis to psychological 
stress in patients with psoriasis. Br J Dermatol 153: 11141120.

26. Buske-Kirschbaum A, Ebrecht M, Kern S, Hellhammer DH, 2006 Endocrine stress responses in TH1-mediated chronic inflammatory skin disease (psoriasis vulgaris) - do they parallel stress-induced endocrine changes in TH2mediated inflammatory dermatoses (atopic dermatitis)? Psychoneuroendocrinology 31: 439-446.

27. Dufour DR, Jubiz WA 2001 Dynamic procedures in Endocrinology, Chapter 241. In: Becker KL (ed) Principles and Practice of Endocrinology and Metabolism, Lippincot, Philadelfia; 2260-2281.

28. Chrousos GP, 1995 The hypothalamic-pituitary-adrenal axis and immune mediated inflammation. $\mathrm{N}$ Engl $\mathrm{J}$ Med
332: 1351-1362.

29. Elenkov IJ, Chrousos GP, 1999 Stress Hormones, Th1/Th2 patterns, pro/anti inflammatory cytokines and susceptibility to disease. Trends Endocrinol Metab 10: 359-368.

30. Chrousos GP, 2000 Stress, chronic inflammation and emotional and physical well-being: Concurrent effects and chronic sequelae. J Allergy Clin Immunol 106: S275291.

31. Griffiths CEM, 2003 The immunological basis of psoriasis. J Eur Acad Dermatol Venereol 17: Supp 2: 1-5.

32. Tagen M, Stiles L, Kalogeromitros D, et al, 2007 Skin Corticotropin-Releasing Hormone Receptor Expression in Psoriasis. J Invest Dermatol 127: 1789-1791. 